\title{
METABOLIC ACTIVITIES OF MONONUCLEOSIDES ON THE ACTIVATION OF LIVER-PYRUVATE OXIDASE SYSTEM RELATING TO THIAMINE DIPHOSPHATE'
}

\author{
AKIO MAEKAWA, TAKATOSHI ESASHI, TAKAO SUZUKI AND \\ YOSHIKAZU SAHASHI ${ }^{2}$ \\ Laboratory of Biochemistry and Nutrition, Department of Agricultural \\ Chemistry, Tokyo University of Agriculture, Setagaya-ku, Chitose, Tokyo
}

(Received July 4, 1963)

In the previous papers $(1,2)$, the possible metabolism of inosinic acid on the activation of thiamine diphosphate (TDP) in liver-pyruvate oxidase system was studied, and the increase in enzyme activity was confirmed by addition of inosine- 5 '-phosphate (IMP), adenosine- $5^{\prime}$-phosphate (AMP) and guanosine- $5^{\prime}$-phosphate (GMP). Especially, in the case of IMP, the addition of inosine or hypoxanthine plus D-ribose (R) was found to accelerate the liver-pyruvate oxidase system, whereas no activation was seen by a single addition of hypoxanthine. In the experiment with hypoxanthine and D-ribose, the reaction products were tested by paper chromatography, and the reappearance of inosine was always detected on the chromatogram.

In the present paper, further investigations were made for elucidation whether pyrimidine mononucleotides activate the enzyme system. The addition of uridine $-5^{\prime}$ phosphate (UMP), or cytidine-5'-phosphate (CMP) was found to show the similar increase in enzyme activity $(3,4)$ and the metabolic fates of the mononucleotides in the reaction mixture were carefully studied by paper chromatography. Further, various other mononucleotides were also tested and the degradation to each corresponding base and D-ribose was always found to occur. On the other hand, the reappearance of the corresponding mononucleosides was noticed when both the base and D-ribose were added.

These findings have led to the conclusion that mononucleosides are converted into the corresponding bases and D-ribose and both the reaction products can also be synthesized to the mononucleosides which can activate the action of thiamine diphosphate in liver-pyruvate oxidase system as follows.

Mononucleotide $\underset{\mathrm{Pi}}{\longrightarrow}$ mononucloside $\leftrightarrows$ base + D-ribose

\section{EXPERIMENTAL AND RESULTS}

\section{Preparation of Washed Liver Homogenate}

Rats previously fed on an ordinary diet were killed, and the liver was rapidly

1 Metabolic Activities of Nucleic Acids in Vitamin Activation. V.

2 前川昭男, 江指隆年, 鈴木隆雄, 佐橋佳一. 
homogenized with a cold $0.25 \mathrm{M}$ sucrose buffer solution. It was further washed with $0.04 \mathrm{M} \mathrm{MgCl}_{2}$ according to Wright (5) as shown in Fig. 1.

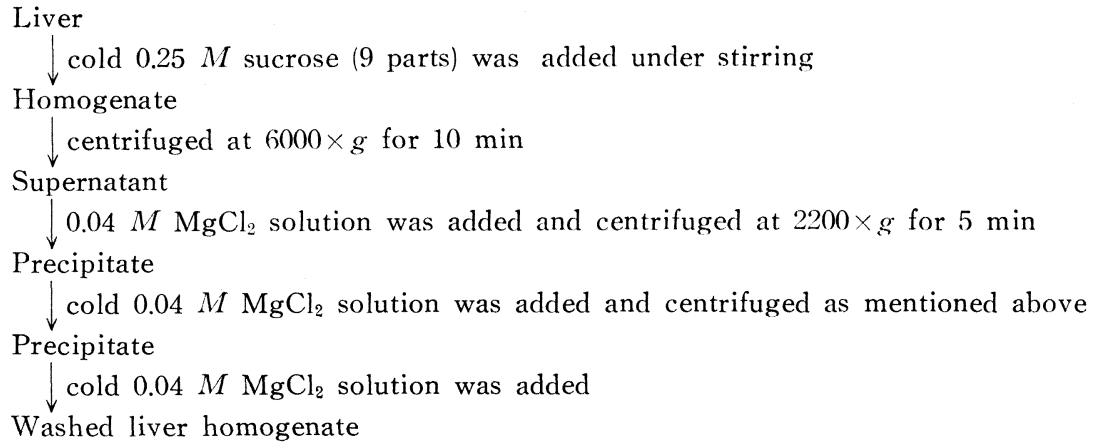

FIt. 1 Preparation of Washed Liver Homogenate

2. Additive Tests of Various Mononucleotides on the Activation in vitro of Liver-Pyruvate Oxidase System

Activities were tested with the modified Wright's pyruvate oxidation system as shown in Table I using various mononucleotides, and the degradation products of the compounds were carefully estimated by the ordinary paper chromatography with the results shown in Figs. 2-3.

3. Additive Effects of Mononucleosides or Bases plus D-Ribose on the Activation of Thiamine Diphosphate in Liver-Pyruvate Oxidase System

Experiments were carried out by the method described above, and the uptake

TABLE I

Reaction System for Pyruvate Oxidation by Rat Liver Homogenate

Washed liver homogenate $\quad 1.0$

$0.001 M$ cytochrome c 0.1

$0.02 M$ nicotinamide-adenine dinucleotide $\quad 0.1$

$0.2 M$ nicotinamide 0.1

$0.02 M$ fumarate 0.1

$0.04 M \mathrm{MgCl}_{2} \quad 0.1$

$1.0 M \mathrm{NaF} \quad 0.1$

$20 \% \mathrm{KOH} \quad 0.4$

$1 / 15 M$ phosphate buffer (pH 7.3) 0.4

$0.01 M$ thiamine diphosphate 0.1

$0.1 M$ sodium pyruvate 0.2

$0.01 M$ adenosine-5' - phosphate $\quad 0.1$

$0.01 M$ guanosine-5' $5^{\prime}$ phosphate 0.1

$0.01 M$ inosine-5'-phosphate 0.1

$0.01 M$ uridine-5'-phosphate 0.1

$0.01 M$ cytidine-5'-phosphate 0.1

$0.01 M$ adenosine or adenine plus D-ribose 0.1

$0.01 M$ guanosine or guanine plus D-ribose 0.1

$0.01 M$ inosine or hypoxanthine plus D-ribose 0.1

$0.01 M$ uridine or uracil plus D-ribose 0.1

$0.01 M$ cytidine or cytosine plus D-ribose 0.1

Water up to 3.0 
of oxygen $\mathrm{Qo}_{2}(\mathrm{~N})$ were estimated by adding the mononuclosides or the corresponding bases. Positive effect was recognized in the incubation of mononucleosides or bases plus D-ribose for the activation of thiamine diphosphate in the enzyme system, while no activity was observed by a single addition of a base. After the experiments, the reaction mixture in the vessel of Warburg's apparatus was analyzed by paper chromatography, and the reappearance of the corresponding mononucleosides was always confirmed (Figs. 4-5).

\section{SUMMARY}

1. The effects of pyrimidine mononucleotides on liver-pyruvate oxidase system was studied, and it was found that the addition of UMP or CMP resulted in similar increase of the enzyme activity. The metabolic fate of the nucleotides in the reaction mixture was quite similar to the case of inosine- 5 '-phosphate.
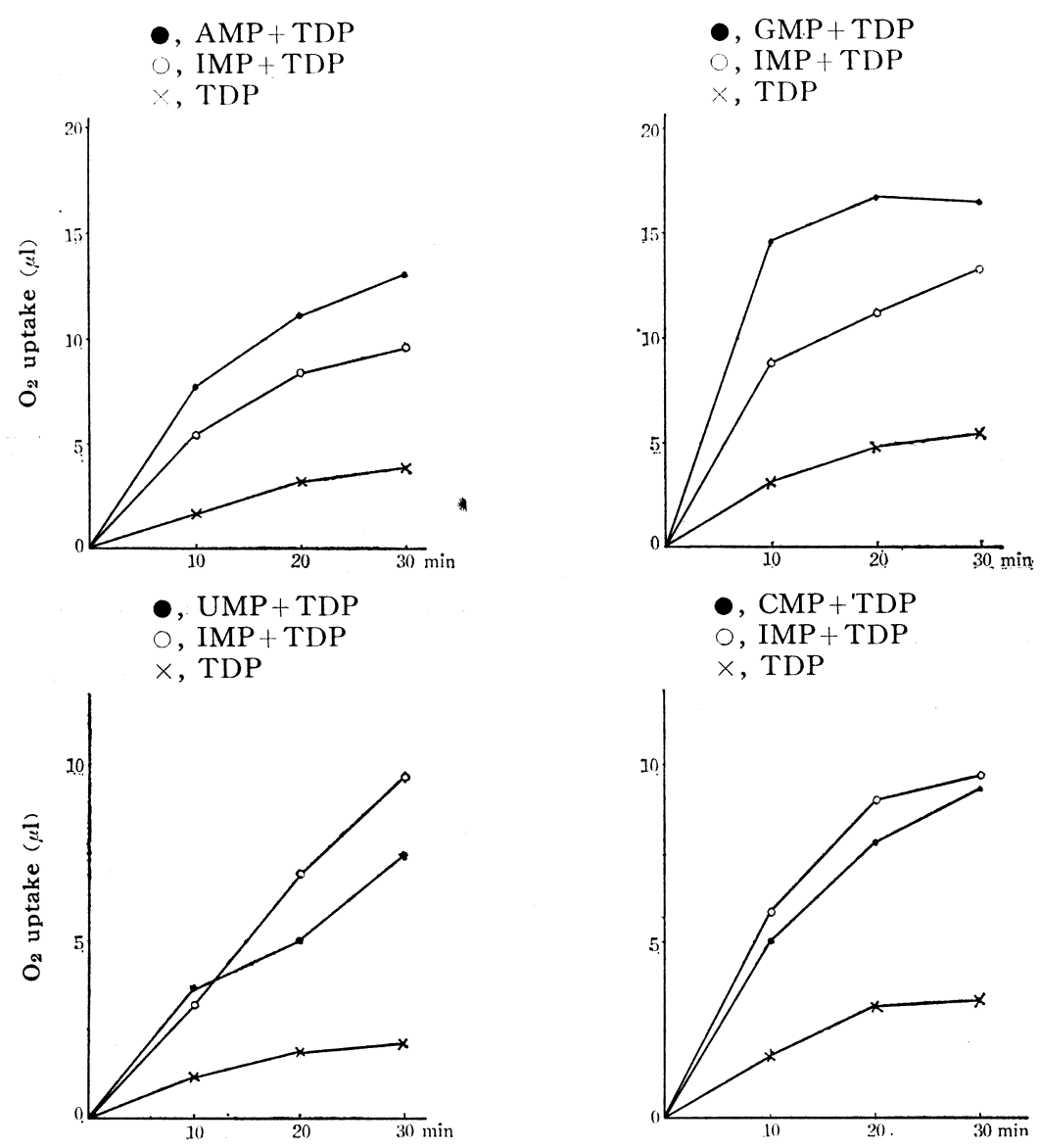

Fir. 2 Effects of Mononucleotides on the Activation of Thiamine Diphosphate in Rat Liver-Pyruvate Oxidase System 

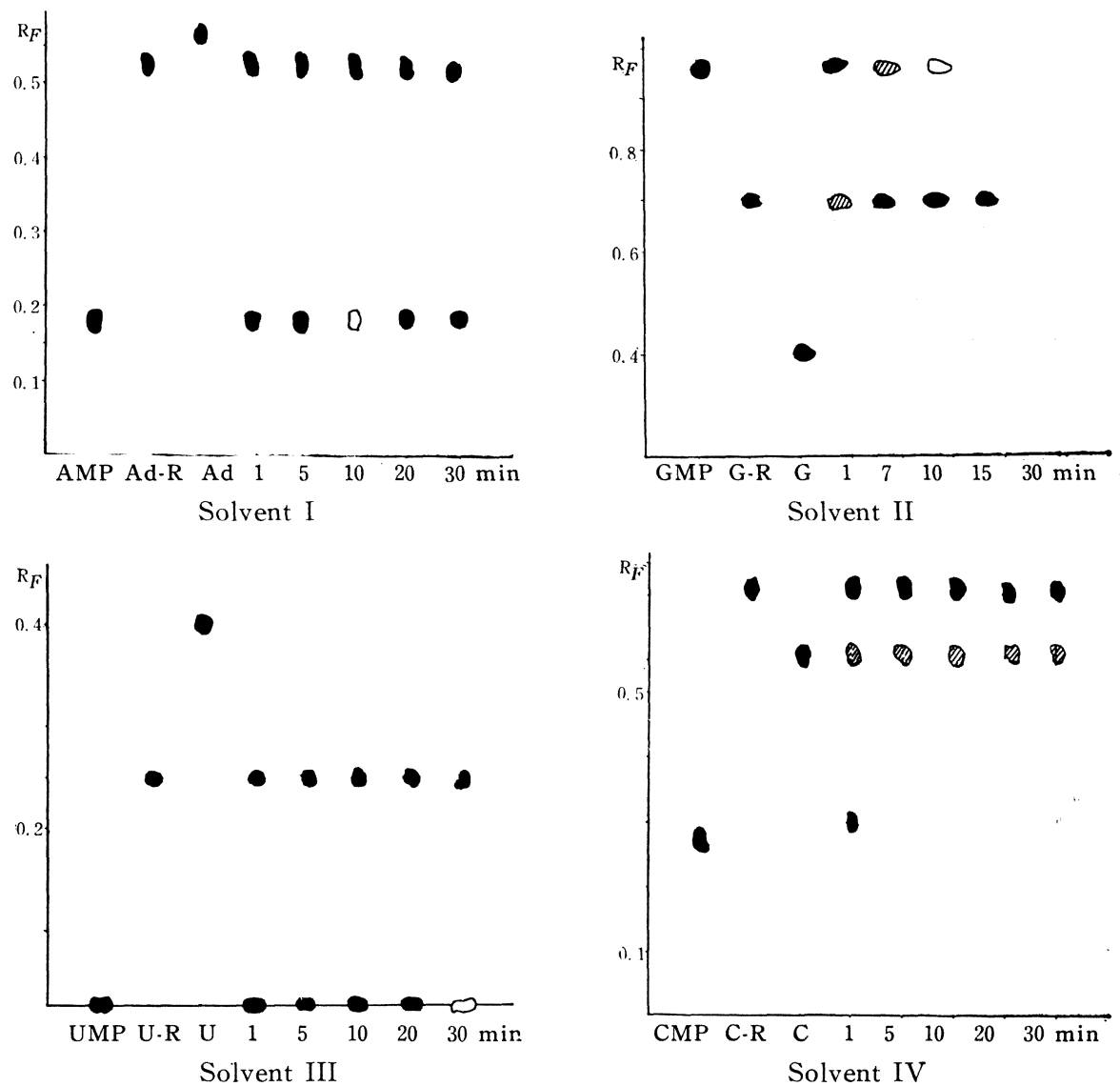

FItx. 3 Paper Chromatograms of the Metabolic Products of Various Mononucleotides in the Reaction Mixture of Rat Liver-Pyruvate Oxidase System

Solvent I, $n$-butanol-acetic acid-water $(12: 3: 5)$; Solvent II, water (pH 5.2 to 5.5); Solvent III, $n$-butanol (water-saturated); Solvent IV, pyridine- $n$-butanol-water $(1: 1: 1)$.

Ad-R, adenosine; Ad, adenine; G-R, guanosine; G, guanine ; I-R, inosine; U-R, uridine ; $\mathrm{U}$, uracil ; C-R, cytidine ; C, cytosine.

2. The effects of various nucleosides on the enzyme activity were also tested, and the degradation products were estimated by paper chromatography. The metabolic fates of the mononucleosides were found to be quite similar to the case of inosine, and the reappearance of the mononucleosides was noticed after addition of both the corresponding bases and D-ribose.

\section{ACKNOWLEDGEMENT}

This work was supported in part by the Vitamin B Research Committee and by the Central Research Laboratory of the Ajinomoto Co., Inc., to which the author's thanks are due 
, AMP + TDP

$\square$, adenosine + TDP

$\triangle$, adenine $+\mathrm{D}$-ribose + TDP

$x$, adenine + TDP

0 , TDP

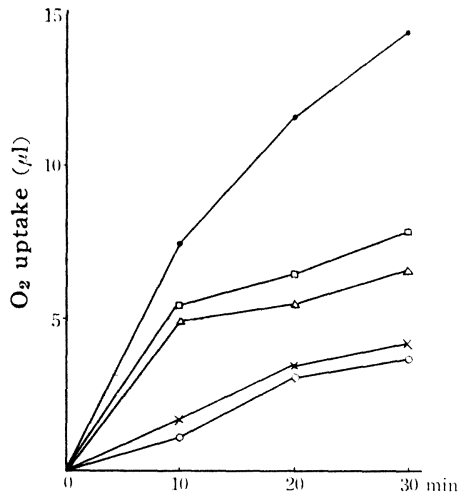

•, UMP + TDP

$\square$, uridine + TDP

$\triangle$, uracil + D-ribose + TDP

$x$, uracil + TDP

o, TDP

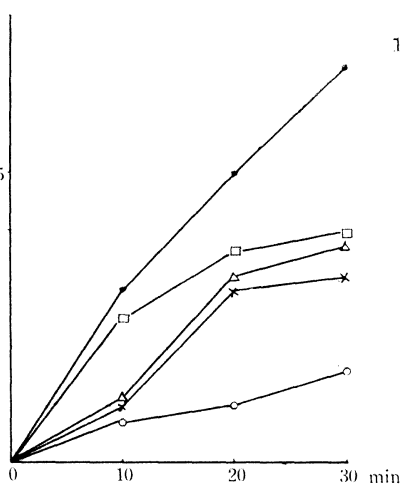

-, $\mathrm{CMP}+\mathrm{TDP}$

$\square$, cytidine + TDP

$\triangle$, cytosine + D-ribose + TDP

$x$, cytosine + TDP

$\mathrm{O}$, TDP

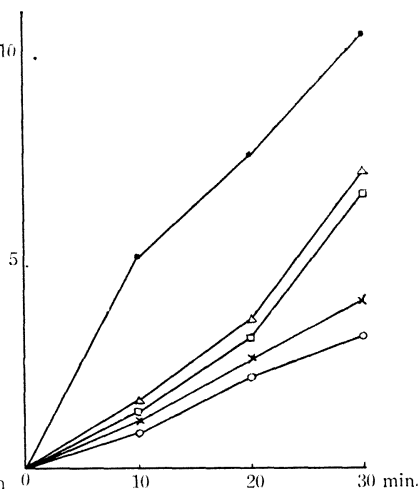

FIg. 4 Effects of Mononucleosides or Bases plus D-Ribose on the Activation of

Thiamine Diphosphate in the Rat Liver-Pyruate Oxidase System
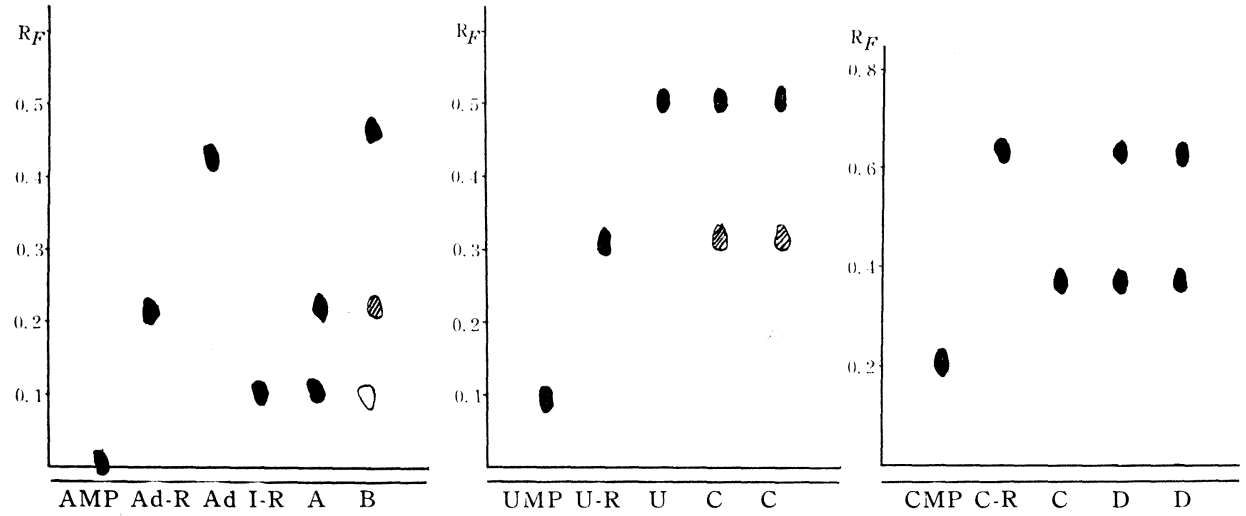

FIa. 5 Paper Chromatograms of the Metabolites of Various Mononucleosides or Bases plus D-Ribose in Rat Liver-Pyrurate Oxidase System
A, reaction products from Ad-R;
$B$, reaction products from $\mathrm{Ad}+\mathrm{R}$;
$\mathrm{C}, \quad, \quad, \quad$ from $\mathrm{U}+\mathrm{R}$;
$\mathrm{D}, \quad, \quad, \quad$ from $\mathrm{C}+\mathrm{R}$.

\section{REFERENCES}

1. Maekawa, A., Hayashi, J., Oshima, T., Suzuki, T., and Sahashi, Y., J. Vitaminol. 7, 271 (1961).

2. Maekawa, A., Suzuki, T., and Sahasih, Y., ibid. 8, 172 (1962).

3. Sahashi, Y., Maekawa, A., Miyazawa, E., and Esashi, T., Vitamins 26, 75 (1962).

4. Sahashi, Y., Maekawa, A., Esashi, T., and Suzuki, T., ibid. 26, 231 (1962).

5. Wright, R.C., and Scott, E.M., J. Biol. Chem. 206, 725 (1954). 